\title{
Radio-frequency plasma potential variations originating from capacitive coupling from the coil antenna in inductively coupled plasmas
}

\author{
M. Watanabe, D. M. Shaw, ${ }^{\text {a) }}$ and G. J. Collins \\ Department of Electrical Engineering, Colorado State University, Fort Collins, Colorado 80523 \\ H. Sugai \\ Department of Electrical Engineering, Nagoya University, Furo-cho, Chikusa-ku, Nagoya, 464-8603, Japan
}

(Received 14 October 1998; accepted for publication 29 December 1998)

\begin{abstract}
The radio-frequency plasma potential in a stove top inductively coupled plasma source is measured by a capacitive probe. The experimental results are compared to a crude circuit model which accounts for capacitive coupling between the rf coil and the bulk plasma. The capacitive coupling model has three terms: the dielectric window capacitance, the sheath capacitance between the dielectric window and the bulk plasma, and the bulk plasma to ground sheath capacitance. The crude circuit model predictions are verified by quantitative comparison with the measured if plasma potential in the bulk argon plasma at pressures from 1 to $20 \mathrm{mTorr}$ and radio-frequency (13.56 $\mathrm{MHz}$ ) plasma power levels from 60 to $1000 \mathrm{~W}$. Finally, the measured ion energy spectrum, as determined by a retarding potential analyzer, agrees with rf plasma potential measurements over the entire range of experimental conditions. (C) 1999 American Institute of Physics. [S0021-8979(99)07507-6]
\end{abstract}

\section{INTRODUCTION}

Inductively coupled plasma (ICP) sources find application in the newest generation of plasma etch tools due to their ability to generate uniform $( \pm 2 \%)$ high density $\left(n_{e}\right.$ $\sim 10^{11} \mathrm{~cm}^{-3}$ ) plasmas at low ambient gas pressure $(p$ $\sim 1-20 \mathrm{mTorr}$ ) over the $20-30 \mathrm{~cm}$ diameter required for ultralarge scale integration (ULSI) wafers. ${ }^{1-3} \mathrm{~A}$ stove top geometry ICP uses an if current passing through an external coil, separated from the plasma by a dielectric window, to apply an azimuthal electric field in the plane parallel to the dielectric window. However, the high rf voltage (up to several $\mathrm{kV}$ ) required to drive the if current through the wide area coil also causes substantial capacitive coupling through the dielectric window from the coil to the plasma via an electric field perpendicular to the window. This rf electric field applied perpendicular to the dielectric window is of practical importance because it makes start-up ignition of the inductively coupled plasma source easier. However, it also tends to cause unwanted sputtering and erosion of the dielectric window over time.

The effects of capacitive coupling on rf plasma potential in a parallel plate rf discharge are well understood. ${ }^{4}$ The circuit model of an ICP, based on an air core transformer, also explains the externally measured electrical parameters in the ICP excitation circuit. ${ }^{5-7}$ These ICP circuit models describe the inductive coupling from the rf coil to the bulk plasma, but assume that capacitive coupling through the dielectric window is negligible. Recently, capacitive power transfer in an ICP has been investigated by adding capacitive coupling from the rf coil to the plasma through the dielectric window to the air core transformer ICP circuit model. ${ }^{8}$ Other researchers have investigated the capacitive coupling be-

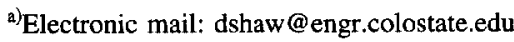

tween a rf biased electrode placed in contact with an ICP and the rf plasma potential, $v_{\mathrm{rf}}{ }^{9}$ but they neglected capacitive coupling from the of coil to the bulk plasma. Herein, we extend the previous ICP circuit model ${ }^{8}$ to better account for capacitive coupling from the high voltage, wide area coil to the bulk plasma, and present experimental observations supporting the combined ICP/capacitive model.

Three capacitors are required in the crude capacitive coupling circuit model: the dielectric window capacitance; the dielectric window-to-bulk plasma sheath capacitance; and the bulk plasma-to-chamber wall sheath capacitance. Sheath capacitance herein is calculated employing the if sheath theory of Godyak and Sternberg, ${ }^{10}$ which provides the rf sheath thickness in a symmetrically driven rf discharge. Herein, we investigate capacitive coupling from the coil to the plasma in an inductively coupled plasma, which is heavily asymmetric. Therefore, it must be noted that many of the assumptions in Ref. 10 may not be suitable. Our model treats the bulk plasma as an equipotential highly conducting body, to a first approximation, which also may introduce further errors. Nevertheless, this simple hybrid circuit model is solved for the expected rf plasma potential, and compared to measured data with good agreement in pressure range from 1 to 20 mTorr.

\section{EXPERIMENTAL APPARATUS AND METHODS}

A schematic of the ICP source used in this experiment is shown in Figure 1. The plasma is excited in a well-grounded aluminum chamber $25 \mathrm{~cm}$ in diameter and $12 \mathrm{~cm}$ high. A 20 $\mathrm{cm}$ diameter, four turn copper coil is placed on top of a 1.4-cm-thick quartz dielectric window to provide inductive coupling to the discharge. A traditional " $L$ " type match network using vacuum capacitors transforms the inductive impedance of the plasma to the $50 \Omega, 13.56 \mathrm{MHz}$ power sup- 


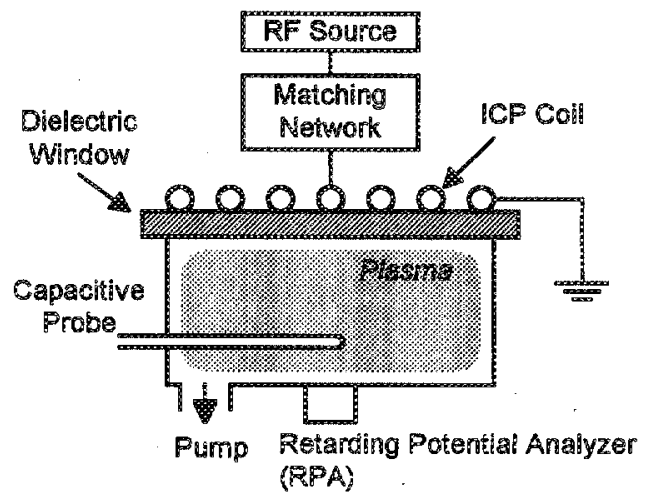

FIG. 1. Schematic diagram of the experimental ICP apparatus including both the If capacitive probe and the retarding potential analyzer (RPA) for measuring ion energy spectra.

ply. Total power delivered from the if power supply to the ICP coll, $P_{\mathrm{rf}}$, is varied from 60 to $1000 \mathrm{~W}$. Herein, we neglect any ohmic power losses in the if coil due to coil resistance. This has no effect on the results presented below since our model for calculating the if plasma potential $v_{\text {rf }}$ uses measured electron density $n_{e}$ and temperature $T_{e}$, and if coil voltage, and does not reiy on plasma power measurements as explained in Sec: IV below. The rf voltage at the input (center) of the coil is measured using a plasma impedance probe (Advanced Energy $\mathrm{RFZ} 60$ ). The accuracy of the RFZ 60 has been independently deternined to be within about $15 \%$ of actual values over the range of parameters in this work. "The RPA shown in Fig. 1 is a retarding potential analyzer for measuring ion energy spectra impinging on the chamber wall as will be more fully discussed in the Appendix.

In the rf plasma potential measurements, argon in the pressure range from 1 to 20 mitor is used as the feedstock gas. Plasma electron density $n_{e}$ and electron temperature $T_{e}$ are independently measured with a if compensated Langmuir probe. Orbital motion limited (OML) theory is used for the plasma electron density calculations. ${ }^{5,12,13}$ The plasma electron temperature is derived from the relation: $V_{p}-V_{f}$ $=\left(T_{e} / 2\right) \ln (M / 2 \pi m)+T_{e} / 2 \approx 5.2 T_{e}$ for argon, where $V_{p}$ is the plasma potential (Volts), $V_{f}$ is the floating potential (Volts), $M$ is the argon mass $\left(40 / 6.02 \times 10^{23}=6.64\right.$ $\times 10^{-23} \mathrm{~kg}$ ), and $m$ is the electron mass $\left(9.11 \times 10^{-31} \mathrm{~kg}\right)$. Typical values are $n_{e}=10^{11} \mathrm{~cm}^{-3}$ and $T_{e}=3.2 \mathrm{eV}$ at $2 \mathrm{mTorr}$ argon and $P_{\mathrm{rf}}=250 \mathrm{~W}$.

The rf plasma potential is measured using a capacitive probe construcked by placing a length of semirigid, $50 \Omega$ coaxial cable inside a Pyrex glass sleeve, ${ }^{14,15}$ as shown in Fig. 2. The center conductor of the coaxial cable is separated from the plasma by the Pyrex glass tube, which forms a

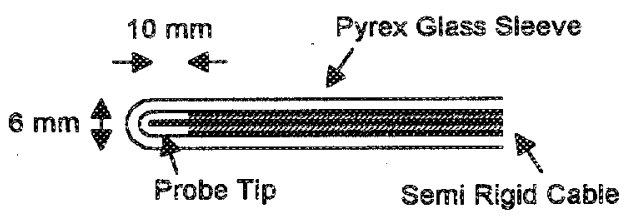

FIG. 2. The geometrical configuration of the capacitive probe.
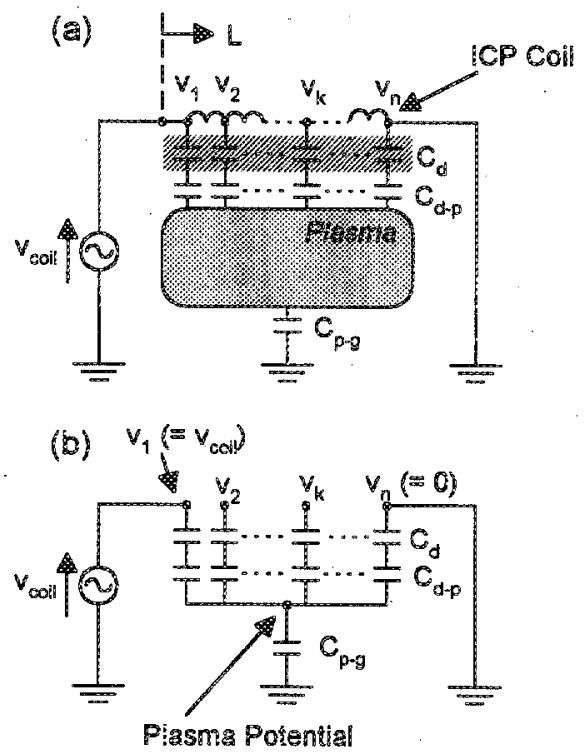

FIG. 3. The spatially distributed capacitance model is shown in two ways. (a) Basic capacitive voltage divider circuit model with the bulk plasma fixed at an equipotential. Here the wide area ICP coil, dielectric window capacitance, and dielectrie wincow-to-bulk plasma sheath capacitance are divided into $n$ distributed parts. (b) Simplified circuit model used for the rf plasma potential calculation.

capacitor. The capacitive probe is terminated in the $50 \Omega$ input of a digital oscilloscope. The capacitive probe is typically placed at a location $8 \mathrm{~cm}$ below the quartz dielectric window and in the center radial position, $r=0$. In order to measure the spatial distribution of the rf plasma potential, the capacitive probe can be moved in the chamber in the $r-z$ plane. The capacitive probe is calibrated by covering the outside of the probe with aluminum foil, applying a known voltage to the foil, and comparing the measured voltage to the known applied voltage. Based on a voltage divider circuit between the capacitive probe impedance and the oscilloscope impedance, we estimate the probe capacitance to be about $4 \mathrm{pF}$.

When the capacitive probe is inserted into the piasma, an additional series capacitance is forned across the plasma-toprobe sheath, which can cause some difference between calibrated and measured results especially since this sheath capacitance varies with plasma conditions. In the measurements discussed below, it is assumed that this sheath capacitance is very large as compared to the probe capacitance, due to the small sheath width, and therefore ignored.

We also rote that the if plasma potential as measured by the capacitive probe has been compared to the ion energy spectrum impinging on the grounded chamber wall as an independent verification of the probe's accuracy. These results are presented in the Appendix.

\section{CIRCUTT MODEL FOR CAPACITIVE COUPLAG}

Capacitive coupling from the external if coil to the buik plasma in an ICP is represented by the three series capacitors which form a voltage divider circuit as shown in Figure 3(a). Here we assume that the coil resistance is negligible and the whole bulk plasma volume is at an equipotential, which 
means that the bulk plasma has negligible impedance. The first capacitor, $C_{d-p}$, represents the if sheath formed between the dielectric window and the bulk plasma. The second capacitor, $C_{p-g}$, is the rf sheath formed between the bulk plasma and the grounded chamber wall. The dielectric window forms the third capacitor, $C_{d}$. Thus, the if voltage from source to ground is placed across the three series capacitors:

$$
v_{\text {coil }}=v_{d}+v_{d-p}+v_{p-g},
$$

where $v_{\text {coil }}$ is the $\mathrm{rf}$ coil voltage with respect to ground, $v_{d}$ is the rf voltage drop across the dielectric window, $v_{d-p}$ is the dielectric window-to-bulk plasma sheath voltage drop, and $v_{p-g}$ is the bulk plasma-to-grounded wall sheath voltage drop. Furthermore, if current continuity for the capacitive circuit in the absence of harmonic currents requires

$$
C_{d} v_{d}=C_{d-p} v_{d-p}=C_{p-g} v_{p-g} .
$$

The rf plasma potential, which is equal to $v_{p-g}$, is found by solving Eqs. (1) and (2) simultaneously.

Numerical calculation of the dielectric window capacitance $C_{d}$ is possible, but difficult, owing to the complicated geometry of the open coil. To experimentally estimate $C_{d}$, an electrically grounded conducting sheet is placed under the dielectric window. This forms a capacitor with the coil and grounded sheet as the two conductors and the dielectric window as the capacitor dielectric. A known capacitor is then placed in series with the formed capacitor, and a low frequency $(f \sim 100 \mathrm{~Hz})$ voltage signal is applied across the series capacitor circuit. The window capacitance is found from the ratio of the voltages measured across the two capacitors. The capacitance of the dielectric window, $C_{d}$ is found by this means to be $13 \mathrm{pF}$. The low frequency test signal is preferred for capacitance determination to minimize the effect of the coil inductance on the measurement, which is large at $13.56 \mathrm{MHz}$.

In estimating the window capacitance as described above, we set the coil at one potential and measure the total capacitance of the dielectric window. However in the actual ICP case, the outside of the coil is grounded, and the rf voltage is applied to the center of the coil. The coil then has a potential distribution along its length from $v_{\text {coil }}$ at the center to $0 \mathrm{~V}$ at the grounded outside terminal. The $13.56 \mathrm{MHz}$ rf potential distribution along the coil is measured by a voltage probe at various points along the coil, and an electrically grounded conducting sheet, which roughly simulates the inductively coupled plasma, is placed under the dielectric window. Figure 4 shows the measured rf potential distribution along the coil, where the horizontal axis, $L$, is the linear distance from the center to the end of the rf coil. One can see that the potential drop along the coil is nonlinear along its length, indicating that the coil impedance is changing with position along the coil. At the center of the coil, the magnetic field produced by the rf current tends to cancel itself out due to the proximity of other turns in the coil, resulting in a lowering of the inductance. On the other hand, near the outside edge of the coil, the magnetic field does not cancel itself out as much, resulting in higher inductance locally near the outside of the coil. It is judged that this is the origin for the

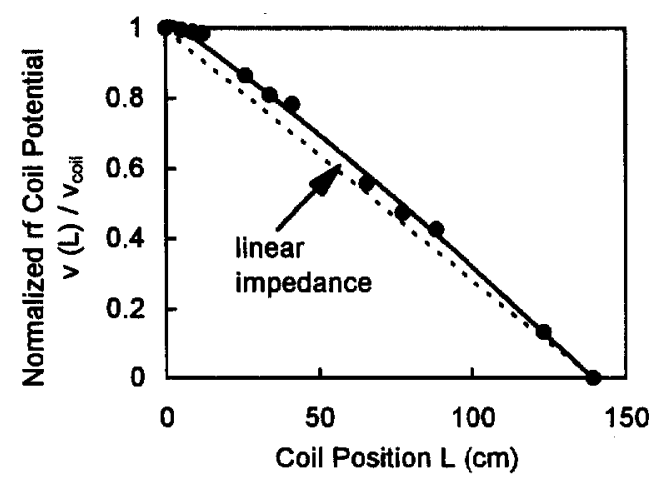

FIG. 4. Normalized rf potential distribution measured along the ICP coil versus position along the coil length $L$.

observed nonlinear voltage drop across the coil. Even if a plasma exists under the dielectric window instead of the electrically grounded conducting sheet, the rf potential distribution still follows the relation in Fig. 4, because the changes in coil impedance, when including the plasma impedance for various plasma conditions, is small enough to neglect.

To account for the distribution of voltage along the rf coil in our model, the coil is divided into " $n$ " parts, ${ }^{8}$ each with a different voltage $v_{k}(k=1,2, \ldots, n)$ as shown in Fig. 3 (b). When $n=1$, the if coil voltage $v_{\text {coil }}$ is applied in series to these three capacitances $C_{d}, C_{d-p}$, and $C_{p-g}$. The rf plasma potential $v_{\text {rf }}^{*}$ is given by

$$
v_{\mathrm{rf}}^{*}=\frac{C_{d} C_{d-p}}{C_{d-p} C_{p-g}+C_{d} C_{p-g}+C_{d} C_{d-p}} \times v_{\text {coil }} .
$$

When $n>1$, the voltage across the first branch $v_{1}$ is equal to $v_{\text {coil }}$ and across the $k$ th branch $v_{k}(k=1,2, \ldots, n)$ is taken from the measured voltage distribution in Fig. 4. Across the $n$th branch $v_{n}$ is equal to $0 \mathrm{~V}$, also as shown in Fig. 4.

The dielectric window capacitance $C_{d}$ and the dielectric window-to-bulk plasma sheath capacitance $C_{d-p}$ are also each broken into $n$ elements as well. These $n$ pairs of series connected $C_{d k}$ and $C_{d-p k}$ capacitors $(k=1,2, \ldots, n)$ are connected in parallel to the common point of the bulk plasma, as shown in Fig. 3(b). In general, the capacitance between two parallel plates, neglecting fringe field, is then expressed as

$$
C=\epsilon_{r} \epsilon_{0} A / d \text {, }
$$

where $\epsilon_{r}$ is the relative permittivity (the relative permittivity is assumed to be $\left.\epsilon_{r}=1\right), \epsilon_{0}$ is the permittivity of free space $\left(\epsilon_{0}=8.85 \times 10^{-12} \mathrm{~F} / \mathrm{m}\right), A$ is the area of the parallel plates $\left(m^{2}\right)$, and $d$ is the separation of the two plates $(m)$. In our model, we use the dielectric window area $A_{d}$ to calculate $C_{d-p}$, and $A_{g}$ as the grounded wall area for $C_{p-g}$.

The capacitances $C_{d k}$ and $C_{d-p k}$ of the $k$ th branch are given as

$$
C_{d k}=C_{d} / n
$$

and

$$
C_{d-p k}=\epsilon_{r} \epsilon_{0} A_{d} / n d_{d-p k}^{\prime} \quad(k=1,2, \ldots, n)
$$

and $C_{p-g}$ is expressed as 


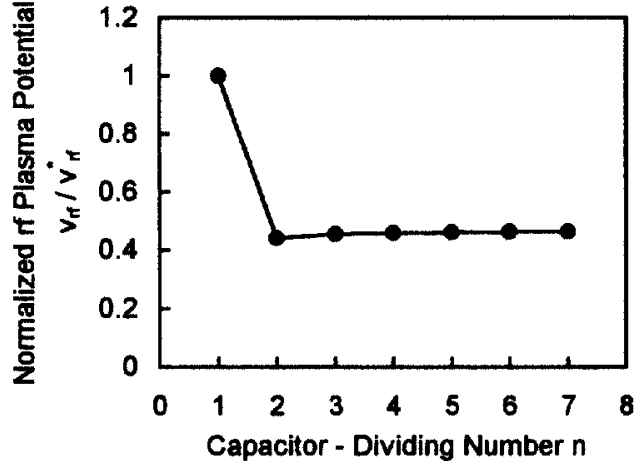

FIG. 5. Normalized rf plasma potential calculated using the spatially distributed capacitive voltage divider circuit model for various integer numbers of lateral capacitors $n$.

$$
C_{p-g}=\epsilon_{r} \epsilon_{0} A_{g} / d_{p-g}^{\prime},
$$

where $d_{d-p k}^{\prime}$ is the "capacitive" dielectric window-to-bulk plasma sheath width, which means the effective plate separation for $C_{d-p k}$, and $d_{p-g}^{\prime}$ is the capacitive bulk plasma-togrounded wall sheath width. These are then calculated from Eq. (51) in Ref. 10 as

$$
d^{\prime}=\lambda_{D} v_{\mathrm{sh}} / T_{e \rho},
$$

where $\lambda_{D}$ is the plasma Debye length $(m)$ at the presheathsheath edge, $v_{\mathrm{sh}}$ is the rf component of the sheath voltage (Volts), $T_{e}$ is the electron temperature (Volts), and $\rho$ is the if sheath oscillation parameter. A variable, $u_{c}^{1}$, can be found in Eq. (51) in Ref. 10, which is defined as the fundamental harmonic of $\mathrm{rf}$ voltage across the two rf sheaths in a symmetric discharge normalized by the electron temperature. We assume that the if voltage across one sheath in our experiment is half of the rf voltage across the two sheaths, therefore $v_{\mathrm{sh}}=u_{c}^{1} T_{e} / 2 . \rho$ is found from Eq. (60) in Ref. 10 for $\rho<1$, which is valid under most of our experimental conditions:

$$
0.3398 \rho^{4}+1.375 \rho^{3}+\delta \rho=v_{\mathrm{sh}} / T_{e},
$$

where $\delta$ is 2.612 for argon, our feedstock gas. Equation (59) in Ref. 10 is more suitable for $\rho>1$ than Eq. (9) above. However Eq. (9) is used for all of our calculations herein to simplify the set of simultaneous equations. The error of the calculated capacitance using Eq. (60) instead of using Eq. (59) is within $3 \%$. Equations (1) and (2) are also modified to satisfy both the Kirchoff voltage and current laws for $n$ branches,

$$
\begin{aligned}
& v_{k}=v_{d k}+v_{d-p k}+v_{p-g} \quad(k=1,2, \ldots, n), \\
& \sum_{k=1}^{n} C_{d k} v_{d k}=\sum_{k=1}^{n} C_{d-p k} v_{d-p k}=C_{p-g} v_{p-g} .
\end{aligned}
$$

In order to solve for the if plasma potential, Eqs. (5)(11) are solved simultaneously. Figure 5 shows the if plasma potential calculated using the above theory as a function of the number of series elements $n$. One can see that the calculated rf plasma potential rapidly converges with increasing $n$. In this work, we use $n=5$ in all calculations.

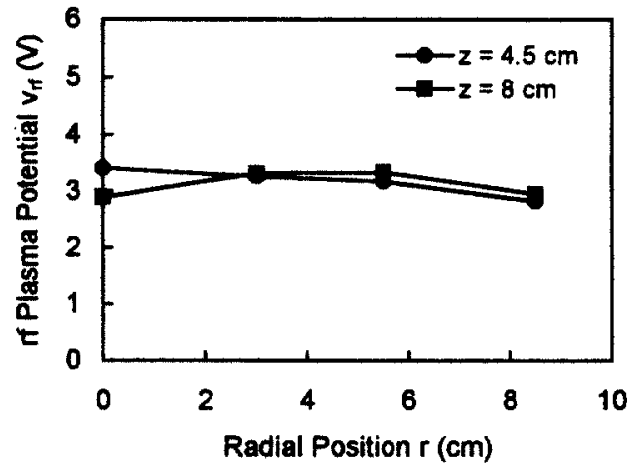

FIG. 6. Radial spatial distribution of the measured if plasma potential at two distances $z$ below the dielectric window: $z=4.5$ (circle) and $8 \mathrm{~cm}$ (square). The absorbed of power is $100 \mathrm{~W}$, in a $20 \mathrm{mTorr}$ argon ambient.

\section{RESULTS AND DISCUSSIONS}

The equipotential plasma body approximation is verified by measuring the spatial distributions of the rf plasma potential. Figure 6 shows radial distributions of rf plasma potential (peak-zero value) at two distinct distances $z$ below the dielectric window: $z=4.5$ and $8 \mathrm{~cm}$. One can see that in 20 mTorr argon, the rf plasma potential is less than $4 \mathrm{~V}$ while coil voltage is very large, in the range of $1000 \mathrm{~V}$, if plasma potential is nearly uniform spatially, indicating that the equipotential bulk plasma assumption is indeed valid.

Figures 7(a)-7(d) show the measured (circle) and theoretical (square) rf plasma potential versus rf power for four pressures in the 1-20 mTorr range of ambient argon feedstock. The theoretical value $v_{\mathrm{rf}}$ is calculated from the measured value of $v_{\text {coil }}, n_{e}$, and $T_{e}$ in the crude model developed in the previous section. Note the decrease in rf plasma potential with both increasing rf power and with increasing pressure. These trends occur for both the measured if plasma potential as well as in the modeled results.

The decrease in if plasma potential with increasing if power can be easily explained by inspection of our crude circuit model. Here for illustration we oversimplify the number of capacitor branches to $n=1$, which leads to a wrong value of the rf plasma potential ( $\sim 2.5$ times larger than that with $n=5$ ), but still maintains almost the same functional relationship between $\mathrm{rf}$ power and $\mathrm{rf}$ plasma potential. For our window thickness, the dielectric window capacitance $C_{d}$ is much less than the dielectric window-to-bulk plasma capacitance $\left(C_{d-p}\right)$ or the bulk plasma-to-grounded wall capacitance $\left(C_{p-g}\right)$, i.e., $C_{d} \ll C_{d-p}$ and $C_{d} \ll C_{p-g}$. Therefore the rf plasma potential $v_{\text {rf }}$ may be approximated as

$$
v_{\mathrm{rf}} \approx v_{\text {coil }}\left(C_{d} / C_{p-g}\right) \text {, }
$$

where $C_{d}$ is constant, so $v_{\text {rf }}$ can be expressed as a function of $v_{\text {coil }}$ and $C_{p-g}$. In our experimental conditions, $v_{\text {coil }}$ increases from 1200 to $2800 \mathrm{~V}$ when delivered of power is increased from 60 to $1000 \mathrm{~W}$ at $2 \mathrm{mTorr}$ argon pressure. On the other hand, $C_{p-g}$ is proportional to $1 / d_{p-g}^{\prime}$ from Eq. (7), where $d_{p-g}^{\prime}$ is the capacitive bulk plasma-to-grounded wall sheath width. From Eqs. (8) and (9) of our article, $d_{p-g}^{\prime}$ is expressed as

$$
d_{p-8}^{\prime}=\lambda_{D}\left(0.3398 \rho^{3}+1.375 \rho^{2}+\delta\right) .
$$



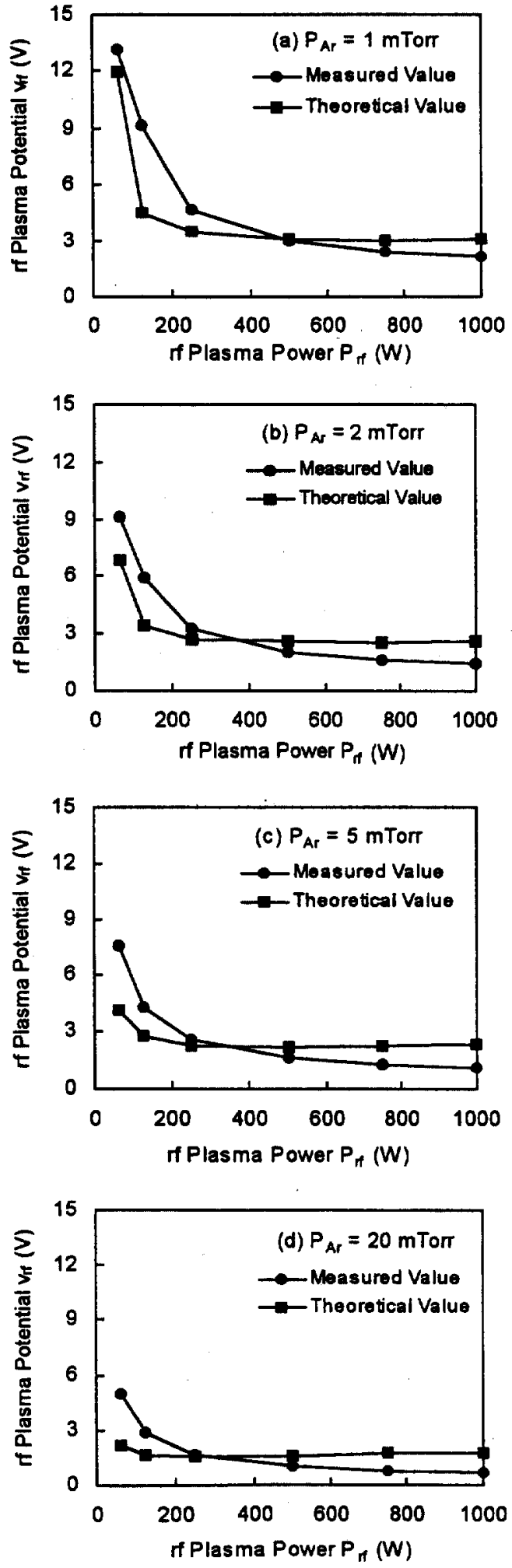

FIG. 7. The if power dependence of both the measured and calculated if plasma potential $V_{\mathrm{rf}}$ in (a) 1, (b) 2, (c) 5, and (d) 20 mTorr of argon, respectively.

$d_{p-g}^{\prime}$ is composed of two factors, the Debye length $\lambda_{D}$ and the factor $\left(0.3398 \rho^{3}+1.375 \rho^{2}+\delta\right) . \lambda_{D}$ varies from 0.194 to $0.032 \mathrm{~mm}$ when rf power is increased from 60 to $1000 \mathrm{~W}$; on the other hand the term $\left(0.3398 \rho^{3}+1.375 \rho^{2}+\delta\right)$ varies only from 3.07 to 2.69 . The capacitive bulk plasma-to-grounded

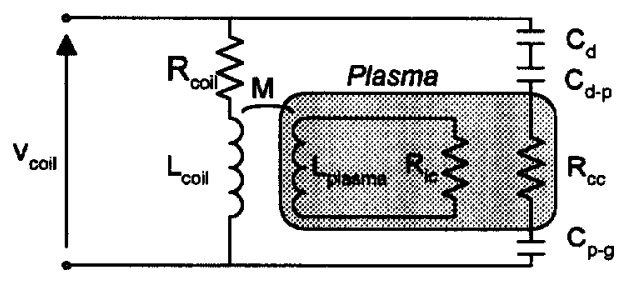

FIG. 8. Air core transformer mutual coupling circuit model for an ICP including the effects of capacitive coupling from the wide area rf coil to the bulk plasma.

wall sheath width $d^{\prime}$ is roughly proportional to the Debye length $\lambda_{D}$, and therefore $C_{p-g}$ is also roughly proportional to $\lambda_{D}$. So $v_{\mathrm{rf}}$ can be simply expressed to first order as

$$
\begin{aligned}
v_{\text {rf }}(\text { plasma potential }) & \approx v_{\text {coil }}\left(C_{d} / C_{p-g}\right) \\
& \approx v_{\text {coil }} C_{d} d_{p-g}^{\prime} / \epsilon_{r} \epsilon_{0} A_{g} \\
\left.v_{\text {rf }} \text { (plasma potential }\right) & \propto v_{\text {coil }} \lambda_{D} .
\end{aligned}
$$

The rf plasma potential is therefore approximately a function of the applied coil voltage $v_{\text {coil }}$, and the Debye length $\lambda_{D} . \lambda_{D}$ is solely a function of the plasma electron density $n_{e}, \lambda_{D}=\left(\epsilon_{0} T_{e} / e n_{e}\right)^{1 / 2}$ since at a constant pressure, $T_{e}$ is roughly constant. ${ }^{5}$ So the if plasma potential is expressed as

$$
v_{\text {rf }} \text { (plasma potential) } \propto v_{\text {coil }} n_{e}^{-1 / 2} .
$$

In general, the plasma electron density $n_{e}$ is nearly proportional to rf power $P_{\text {rf }}$ (Ref. 5) if we neglect ohmic coil losses. This is why the rf plasma potential decreases in our model as well as in practice with increasing rf power. In our experimental conditions, $v_{\text {coil }}$ increases only 2.3 times while $P_{\text {rf }}$ changes from 60 to $1000 \mathrm{~W}$, indicating that the rf plasma potential $v_{\mathrm{rf}}$ is more strongly influenced by $P_{\mathrm{rf}}$

Figure 8 shows the conventional air core transformer circuit model for inductive coupling to an ICP with our crude capacitive coupling model of the wide area coil added, which consists of three capacitors and one resistor. ${ }^{8}$ The series combination of the dielectric window capacitance $C_{d}$, the plasma resistance representing the capacitively coupled power dissipation, $R_{c c}$, and the two sheath capacitances $C_{d-p}$ and $C_{p-g}$ are added in parallel to the air core transformer model, where $R_{\text {coil }}$ is coil resistance, $L_{\text {coil }}$ is the coil inductance, and $L_{\text {plasma }}$ is the bulk plasma inductance. $L_{\text {coil }}$ and $L_{\text {plasma }}$ are magnetically coupled by the mutual inductance term, $M$. The two resistances $R_{i c}$ (representing inductive power transfer) and $R_{c c}$ (representing capacitive power transfer) are primarily a function of electron density. In the low electron density region, most of the power transfer to the plasma is capacitive in nature and $R_{c c}$ dominates the power consumption in the low power plasma. As plasma density increases, more power is transferred inductively, and $R_{i c}$ tends to dominate. In case that the sheath reactance $\left(1 / \omega C_{d-p}\right)$ and plasma resistance in capacitive coupling $\left(R_{c c}\right)$ are small enough to neglect, $v_{\text {rf }}$ is determined simply by the ratio of two capacitances (the dielectric window capacitance and the bulk plasma-to-grounded wall sheath ca- 
pacitance) and $v_{\text {coil }}$. The plasma potential $v_{\text {rf }}$ decreases with increasing plasma density, as mentioned above. Even if the if plasma potential $v_{\mathrm{rf}}$ is small in the high electron density plasma case, a considerable amount of rf current flows through the capacitive portion of the circuit model, robbing the ideal transformer of primary current, which means that the coil voltage still influences the plasma via capacitive coupling. In order to remove the capacitive coupling in the primary from the transformer circuit, a device such as a Faraday shield has been recommended. ${ }^{16,17}$

\section{CONCLUSION}

A crude capacitive voltage divider model has been developed to investigate the relative role of capacitive coupling from the high voltage if excitation coil to the bulk plasma in an ICP as we vary the input if power. The values for sheath capacitances were estimated from the sheath model of Godyak and Sternberg. ${ }^{10}$ The calculated if plasma potential shows good agreement with measured results in the pressure region from 1 to 20 mTorr. The crude circuit model shows that in the low power region, the rf plasma potential is large, while at the higher power range, the rf plasma potential is substantially reduced. In an ICP source, the if plasma potential is mainly dependent on the rf coil voltage, the dielectric window capacitance, and the bulk plasma-to-grounded wall sheath capacitance.

\section{ACKNOWLEDGMENTS}

This work was performed at Colorado State University during a cooperative CSU-Nagoya University research visit of one of the authors (M.W.), and was supported in part by NSF (DMI-970292), NEDO, and Advanced Energy Industries.

\section{APPENDIX}

The ion energy distribution at a grounded surface facing the bulk if plasma is related to the if plasma potential. We derive the relation between the two parameters and verify that the capacitive probe is an accurate tool for measuring the rf plasma potential in an ICP source.

In the case of a collisionless sheath, an ion traversing the sheath gains an energy related to the difference between the instantaneous value of the plasma potential and the potential of the surface it impinges on. If the plasma potential has an $\mathrm{rf}$ component, then the ion energy distribution (IED) will have some energy spread, $\Delta E(\mathrm{eV})$. This energy spread may be estimated as follows: ${ }^{18,19}$

$$
\Delta E \sim 8 v_{\mathrm{rf}}\left(2 e V_{\mathrm{dc}} / M\right)^{1 / 2} / 3 d_{\mathrm{sh}} \omega,
$$

where $d_{\mathrm{sh}}$ is sheath width $(m), \omega$ is the rf excitation frequency $(\mathrm{rad} / \mathrm{s}), M$ is the ion mass $(\mathrm{kg}), V_{\mathrm{dc}}$ and $v_{\mathrm{rf}}$ are the dc and rf components of the plasma potential (Volts), respectively.

In the case of a collisionless sheath, we can use Eq. (61) in Godyak and Sternberg ${ }^{10}$ to calculate the sheath width $d_{\text {sh }}$, which is different from the capacitive bulk plasma-togrounded wall sheath width $d_{p-g}^{\prime}$.

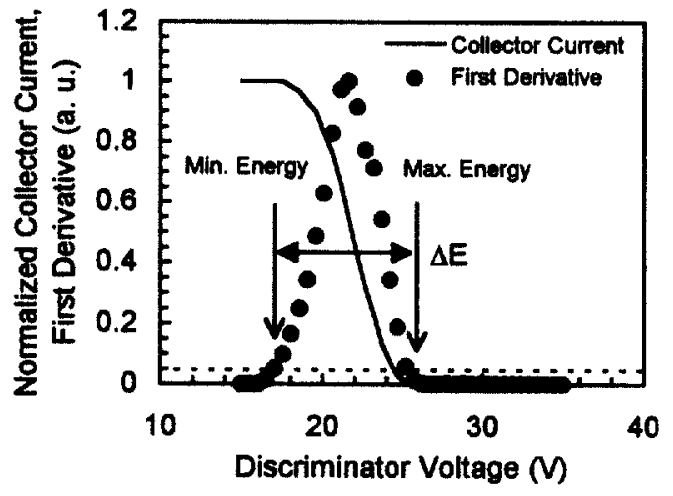

FIG. 9. Normalized ion collector current and first derivative vs discriminator voltage as measured by a retarding potential analyzer (RPA) for the ICP conditions of if power of $125 \mathrm{~W}$ and $2 \mathrm{mTorr}$ argon. $\Delta E$ is the energy spread of incident argon ions from the bulk plasma to the sheath.

$$
d_{\mathrm{sh}}=\lambda_{D}\left\{\delta+\rho+2 \rho^{2}+(5 / 12) \rho^{3}\right\},
$$

where $\rho$ is if sheath oscillation parameter and $\delta$ is 2.612 in argon feedstock gas as described in the body of this article. The Debye length $\lambda_{D}$ is calculated from the measured plasma electron density and electron temperature. The $\mathrm{dc}$ plasma potential in Eq. (A1) is taken from knee of the experimental Langmuir probe current-voltage $(I-V)$ characteristic. The ion energy spread $\Delta E$ is taken from the measured ion energy distribution using a retarding potential analyzer (RPA) $)^{19-22}$ placed as shown in Fig. 1. The if plasma potential $v_{\mathrm{rf}}$ is calculated from Eq. (A1).

The differentially pumped RPA is separated from the main chamber by a grounded orifice $200 \mathrm{~mm}$ in diameter and $100 \mathrm{~mm}$ thick. The RPA consists of two grids, the electron repeller grid and the ion energy discriminator grid, and Faraday cup ion collector. The electron repeller and ion energy discriminator grids are made of 304 stainless steel mesh (100 lines $/ \mathrm{cm}$, transmission $T=$ [open area] $/[$ total area] $35 \%$ ). Dimensions of the RPA are $2.4 \mathrm{~mm}$ between the grounded orifice and the electron repeller grid, $9.5 \mathrm{~mm}$ between the electron repeller grid and the ion energy discriminator grid, and $0.8 \mathrm{~mm}$ between the ion energy discriminator grid and the opening in the Faraday cup ion collector. Both the electron repeller grid and the Faraday cup ion collector are negatively biased $(\sim-40 \mathrm{~V})$. The potential of the discriminator grid is swept from 0 to $+60 \mathrm{~V}$ and current on the collector

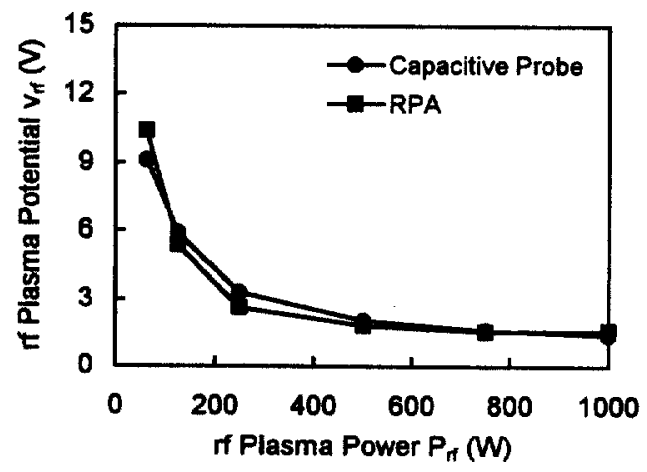

FIG. 10. Rf power dependence of the rf plasma potential as measured separately by both the capacitive probe and the RPA, in 2 mTorr argon. 
electrode is measured. The ion energy distribution is calculated from the derivative of the $I_{c}-V_{d}$ plot, in standard fashion. The resolution of the RPA is estimated to be a few $\mathrm{eV}$ in our experimental conditions. ${ }^{22}$ Figure 9 shows a typical measured $I_{c}-V_{d}$ plot (solid line) and the IED calculated from $d I_{c} / d V_{d}$ (filled circle). The minimum energy and the maximum energy are arbitrarily defined as the energies greater than $5 \%$ of the peak current. The energy spread $\Delta E$ is the difference between the minimum energy and maximum energy.

Figure 10 shows the rf plasma potentials measured by both capacitive probe (filled circle) and RPA (filled square). One can see that these two independent results are in good agreement in spite of using two very different measurement techniques.

' J. H. Keller, J. C. Forster, and M. S. Barnes, J, Vac. Sci. Technol. A 11, 2487 (1993).

${ }^{2}$ J. H. Keller, 42nd Gaseous Electronics Conference, Palo Alto, CA (1989), Paper No. QA-5 (unpublished).

${ }^{3}$ J. H. Keller, M. S. Barnes, and J. C. Forster, 43rd Gaseous Electronics Conference, Champaign-Urbana, IL (1990), Paper No. NA-5 (unpublished).

${ }^{4}$ S. Rauf and M. J. Kushner, J. Appl. Phys. 83, 5087 (1998).

${ }^{5}$ M. A. Lieberman and A. J. Lichtenberg, Principles of Plasma Discharges and Materials Processing (Wiley, New York, 1994).
${ }^{6}$ V. A. Godyak, R. B. Piejak, and B. M. Alexandrovich, Plasma Sources Sci. Technol. 3, 169 (1994).

${ }^{7}$ R. B. Piejak, V. A. Godyak, and B. M. Alexandrovich, Plasma Sources Sci. Technol. 1, 179 (1992).

${ }^{8}$ K. Suzuki, K. Nakamura, H. Ohkubo, and H. Sugai, Plasma Sources Sci. Technol. 7, 13 (1998).

${ }^{9}$ P. A. Miller and M. E. Riley, J. Appl. Phys. 82, 3689 (1997).

${ }^{10}$ V. A. Godyak and N. Sternberg, Phys. Rev. A 42, 2299 (1990).

${ }^{11}$ D. M. Shaw, M. Watanabe, and G. J. Collins (unpublished).

${ }^{12}$ V. A. Godyak, R. B. Piejak, and B. M. Alexandrovich, J. Appl. Phys. 73, 3657 (1993).

${ }^{13}$ I. D. Sudit and R. C. Woods, J. Appl. Phys. 76, 4488 (1994).

${ }^{14}$ N. Benjamin, Rev. Sci. Instrum. 53, 1541 (1982).

${ }^{15}$ M. Yatsuzuka, K. Morishita, K. Satoh, and S. Nobuhara, Jpn. J. Appl. Phys., Part 1 24, 1724 (1985).

${ }^{16}$ H. Sugai, K. Nakamura, and K. Suzuki, Jpn. J. Appl. Phys., Part 1 33, 2189 (1994).

${ }^{17}$ L. J. Mahoney, A. E. Wendt, E. Barrios, C. J. Richards, and J. L. Shohet, J. Appl. Phys. 76, 2041 (1994).

${ }^{18}$ Y. Okamoto and H. Tamagawa, J. Phys. Soc. Jpn. 29, 187 (1970).

${ }^{19}$ J. Liu, G. L. Huppert, and H. H. Sawin, J. Appl. Phys. 68, 3916 (1990).

${ }^{20} \mathrm{~J}$. R. Woodworth, M. E. Riley, P. A. Miller, G. A. Hebner, and T. W. Hamilton, J. Appl. Phys. 81, 5950 (1997).

${ }^{21}$ J. Mathew, R. A. Meger, R. F. Fernsler, and J. A. Gregor, Rev. Sci. Instrum. 67, 2818 (1996).

${ }^{22}$ B. E. Thompson, K. D. Allen, A. D. Richards, and H. H. Sawin, J. Appl. Phys. 59, 1890 (1996). 